\title{
Process optimization for enhancing production of cis-4-hydroxy-L-proline by engineered Escherichia coli
}

\author{
Kequan Chen, Yang Pang, Bowen Zhang, Jiao Feng, Sheng Xu, Xin Wang ${ }^{*}$ and Pingkai Ouyang
}

\begin{abstract}
Background: Understanding the bioprocess limitations is critical for the efficient design of biocatalysts to facilitate process feasibility and improve process economics. In this study, a proline hydroxylation process with recombinant Escherichia coli expressing L-proline cis-4-hydroxylase (SmP4H) was investigated. The factors that influencing the metabolism of microbial hosts and process economics were focused on for the optimization of cis-4-hydroxy-L-proline (CHOP) production.

Results: In recombinant E. coli, SmP4H synthesis limitation was observed. After the optimization of expression system, CHOP production was improved in accordance with the enhanced $\mathrm{SmP4H}$ synthesis. Furthermore, the effects of the regulation of proline uptake and metabolism on whole-cell catalytic activity were investigated. The improved CHOP production by repressing putA gene responsible for L-proline degradation or overexpressing L-proline transporter putP on CHOP production suggested the important role of substrate uptake and metabolism on the whole-cell biocatalyst efficiency. Through genetically modifying these factors, the biocatalyst activity was significantly improved, and CHOP production was increased by twofold. Meanwhile, to further improve process economics, a two-strain coupling whole-cell system was established to supply co-substrate (a-ketoglutarate, $\mathrm{a}-\mathrm{KG}$ ) with a cheaper chemical L-glutamate as a starting material, and $13.5 \mathrm{~g} / \mathrm{L}$ of CHOP was successfully produced.
\end{abstract}

Conclusions: In this study, SmP4H expression, and L-proline uptake and degradation, were uncovered as the hurdles for microbial production of CHOP. Accordingly, the whole-cell biocatalysts were metabolically engineered for enhancing CHOP production. Meanwhile, a two-strain biotransformation system for CHOP biosynthesis was developed aiming at supplying a-KG more economically. Our work provided valuable insights into the design of recombinant microorganism to improve the biotransformation efficiency that catalyzed by Fe(II)/a-KG-dependent dioxygenase.

Keywords: cis-4-Hydroxy-L-proline, Process limitations, Two-strain coupling system

\section{Background}

The microbial production of chemicals and materials has gained increasing attentions as the concerns on environmental problems and limited availability of fossil resource $[1,2]$. More and more bioprocesses have been successfully developed to produce chemicals, fuels, or polymers with metabolically engineering microorganisms $[3,4]$. Hydroxyprolines, such as trans-4-hydroxy-L-proline

\footnotetext{
*Correspondence: wangxin6366@126.com

State Key Laboratory of Materials-Oriented Chemical Engineering,

College of Biotechnology and Pharmaceutical Engineering, Nanjing Tech

University, Nanjing 211816, Jiangsu, China
}

(THOP), and cis-4-hydroxy-L-proline (CHOP), are useful materials for pharmaceutical and cosmetic applications $[5,6]$. Among them, CHOP could inhibit the collagen synthesis and its extracellular deposition as an analogue of L-proline, thereby reducing the growth of tumors [7]. In some bacteria, CHOP is directly produced via fermentation process and the related enzymes in CHOP synthetic pathway have been identified $[8,9]$. Although the product titer is extremely low, these findings show the possibility of utilizing biological processes to produce CHOP.

Two L-proline cis-4-hydroxylases, which belong to the $\mathrm{Fe}(\mathrm{II}) / 2$-oxoglutarate-dependent dioxygenase 


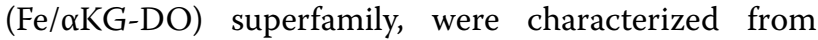
Sinorhizobium meliloti $(\mathrm{SmP} 4 \mathrm{H})$ and Mesorhizobium loti $(\mathrm{MlP} 4 \mathrm{H})$ [8]. These enzymes could catalyze the hydroxylation of L-proline at the cis-4-position to synthesize $\mathrm{CHOP}$ in the presence of $\alpha$-ketoglutarate $(\alpha-K G)$, oxygen and ferrous ion $\left(\mathrm{Fe}^{2+}\right.$ ) (Fig. 1) [8]. When SmP4H was expressed in $E$. coli, only $0.46 \pm 0.15 \mu \mathrm{mol} / \mathrm{g}_{\text {wet cell weight }}$ $\mathrm{CHOP}$ was produced through the fermentation process [5]. Yi et al. reported that the availability of intracellular L-proline was strictly regulated to very low level in the wild type E. coli [10]. Meanwhile, the major generating pathway of $\alpha-K G$, TCA cycle, was also tightly regulated during the L-proline hydroxylation process [10]. In addition, the compound $\mathrm{CHOP}$ is toxic and inhibits the growth of E. coli [5]. Thus, the efficient production of $\mathrm{CHOP}$ via direct fermentation is challenged by not only the complex metabolic engineering manipulation for the improving the supply of precursor L-proline and co-substrate $\alpha-K G$, but also the low $\mathrm{CHOP}$ resistance.

The whole-cell biotransformation might be an alternative approach for CHOP bioproduction. Shibasaki et al. has already established the whole-cell process for the production of several hydroxyprolines, such as THOP and cis-3-hydroxy-L-proline [11]. However, the wholecell process for CHOP production has never been investigated. When using whole-cell biocatalysts, numerous factors could potentially interrupt the catalytic performance, such as expression system, substrate uptake, cosubstrate supply, substrate or product degradation by host endogenous metabolism, and product toxicity [10, 12]. The limiting factors for the effective CHOP bioproduction process remained largely uncharacterized.

In this study, we focused on investigating the factors that might influence the whole-cell biocatalyst efficiency for CHOP production (e.g., expression system, reaction condition, L-proline uptake, L-proline consumption by endogenous metabolism, and $\alpha-K G$ supply).
Furthermore, the CHOP production process was optimized. Meanwhile, a two-strain coupling whole-cell biotransformation system was established for $\mathrm{CHOP}$ biosynthesis.

\section{Methods}

\section{Bacterial strains and media}

All bacterial strains used in this study are listed in Additional file 1: Table S1. Bacteria were grown in Luria-Ber$\operatorname{tani}(\mathrm{LB})$ broth $(10 \mathrm{~g} / \mathrm{L}$ peptone, $5 \mathrm{~g} / \mathrm{L}$ yeast extract and $5 \mathrm{~g} / \mathrm{L}$ sodium chloride) or on LB agar (10 g/L peptone, $5 \mathrm{~g} / \mathrm{L}$ yeast extract, $5 \mathrm{~g} / \mathrm{L}$ sodium chloride and $10 \mathrm{~g} / \mathrm{L}$ agar). Ampicillin (100 mg/L), chloramphenicol $(15 \mathrm{mg} / \mathrm{L})$, streptomycin $(40 \mathrm{mg} / \mathrm{L})$ or kanamycin $(50 \mathrm{mg} / \mathrm{L})$ was added when required.

\section{Plasmid construction}

The plasmids constructed in this study were listed in Table 1. L-Proline cis-4-hydroxylase from Sinorhizobium meliloti NBRC $14782^{\mathrm{T}}$ was codon-optimized for E. coli expression (http://www.jcat.de/) and synthesized by Genewiz (Suzhou, China). Detailed sequence information was provided in Additional file 1: Table S2. Gene $\mathrm{SmP} 4 \mathrm{H}$ was subcloned into $\mathrm{NdeI} / \mathrm{XhoI}$ sites of plasmid pACYCDuet-1, pCDFDuet-1, pRSFDuet-1, pET28a, pET22b and pETDuet-1 to give plasmid pACYCDuet1-SmP4H, pCDFDuet-1-SmP4H, pRSFDuet-1-SmP4H, pET28a-SmP4H, pET22b-SmP4H and pETDuet1-SmP4H respectively (Additional file 1: Figure S1). Gene putP was PCR amplified from E. coli BL21(DE3) genome and ligated to into plasmid pACYCDuet-1 to generate plasmid pACYCDuet-putP. Then the fragment of $\mathrm{P}_{\mathrm{T} 7}$ :putP was PCR amplified with pACYCDuet-putP as template and ligated into XhoI site to give pET28aSmP4H-putP. The L-glutamate oxidase (LOGX) from Streptomyces ghanaensis ATCC14672 was codon-optimized for $E$. coli expression and synthesized by Genewiz.

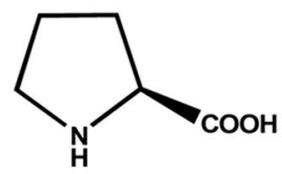

L-Proline<smiles>O=C(O)CCC(=O)C(=O)O</smiles>

a-Ketoglutarate

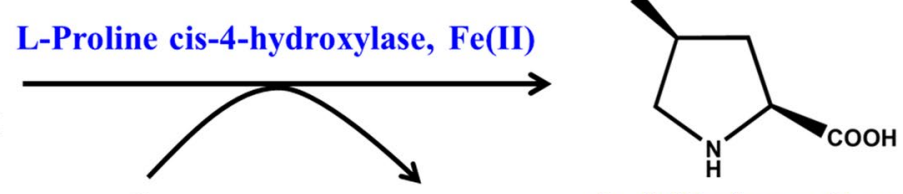

$\mathbf{O}_{2}$ $\mathrm{CO}_{2}$

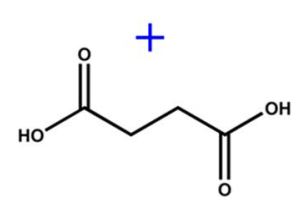

Succinate

Fig. 1 Scheme of cis-4-hydroxy-L-proline (CHOP) synthesis catalyzed by recombinant E. coli expressing L-proline cis-4-hydroxylase 
Table 1 Plasmids used in this study

\begin{tabular}{|c|c|c|}
\hline Plasmids & Description & Source \\
\hline pACYCDuet-1 & Expression vector, $\mathrm{Cm}^{\mathrm{R}}, \mathrm{P}_{\mathrm{T} 7}, \mathrm{P} 15 \mathrm{~A}$ ori & Novagen \\
\hline pCDFDuet-1 & Expression vector, $\mathrm{Sm}^{\mathrm{R}}, \mathrm{P}_{\mathrm{T} 7}, \mathrm{CloDF} 13$ ori & Novagen \\
\hline pRSFDuet-1 & Expression vector, $\mathrm{Km}^{\mathrm{R}}, \mathrm{P}_{\mathrm{T} 7}, \mathrm{RSF}$ ori & Novagen \\
\hline pET28a & Expression vector, $\mathrm{Km}^{\mathrm{R}}, \mathrm{P}_{\mathrm{T} 7}, \mathrm{pBR} 322$ ori & Novagen \\
\hline pET22b & Expression vector, $A m p^{R}, P_{T 7}, p B R 322$ ori & Novagen \\
\hline pETDuet-1 & Expression vector, $A m p^{R}, P_{T 7}, p B R 322$ ori & Novagen \\
\hline pCDF303 & Expression vector, $\mathrm{Sm}^{\mathrm{R}}, \mathrm{P}_{\mathrm{Tr}}, \mathrm{pCDF}$ ori & \\
\hline pACYCDuet-1-SmP4H & Gene SmP4H inserted into Ndel/Xhol sites of pACYCDuet-1 & This study \\
\hline pCDFDuet-1-SmP4H & Gene SmP4H inserted into Ndel/Xhol sites of pCDFDuet-1 & This study \\
\hline pRSFDuet-1-SmP4H & Gene SmP4H inserted into Ndel/Xhol sites of pRSFDuet-1 & This study \\
\hline pET28a-SmP4H & Gene SmP4H inserted into Ndel/Xhol sites of pET28a & This study \\
\hline pET22b-SmP4H & Gene SmP4H inserted into Ndel/Xhol sites of pET22b & This study \\
\hline pETDuet-1-SmP4H & Gene SmP4H inserted into Ndel/Xhol sites of pETDuet-1 & This study \\
\hline pACYC-dCas9 & Gene dCas9 inserted into Ncol/Avrll sites of pACYCDuet-1 & This study \\
\hline pCDF-anti-putA & anti-putA (high) sgRNA sequences inserted into EcoRI/BamHI sites of pCDF303 & This study \\
\hline pACYC-putP & Gene putP inserted into Ncol/Xhol sites of pACYCDuet-1 & This study \\
\hline pET28a-SmP4H-putP & Gene putP inserted into Xhol sites of pET28a-SmP4H & This study \\
\hline pET28a-LOGX & Gene LOGX inserted into Ndel/Xhol sites of pET28a & This study \\
\hline
\end{tabular}

The NdeI/XhoI-digested LOGX fragment was inserted to the $N d e \mathrm{I} / X h o$ I sites of $\mathrm{pET} 28$ a to result in plasmid pET28a-LOGX.

\section{Construction of CRISPRi system to repress gene putA}

A catalytically dead Cas9 mutant (dCas9) derived from Streptococcus pyogenes [13] was synthesized by Genewiz (Suzhou, China) based on the pdCas9-bacterial plasmid (Addgene; Plasmid \#44249), which contains a gene encoding dCas9 protein. NcoI/HindIII-digested dCas9 fragment was ligated into plasmid pACYCDuet-1. The sgRNA chimera, which contains four functional domains including a $\operatorname{Trc}$-inducible promoter, a $20 \mathrm{bp}$ complementary region for binding putA DNA, a $42 \mathrm{bp}$ dCas9-binding hairpin and a $40 \mathrm{bp}$ transcription terminator was synthesized by Genewiz (Nanjing, China) and inserted into EcoRI/BamHI sites of pCDFDuet-1 to give plasmid pCDF-anti-putA (Additional file 1: Table S2). The plasmid pACYC-dCas9 and pCDF-anti-putA were co-transformed into E. coli BL21(DE3) to repress putA expression. PutA activity in crude extracts was determined by measuring $\mathrm{P} 5 \mathrm{C}$ production (nmol $\mathrm{P} 5 \mathrm{C} / \mathrm{min}$ / $\mathrm{mg}$ protein) using o-aminobenzaldehyde as previously described [14].

\section{Cultivation of microorganisms}

The recombinant strain was inoculated from a freshly transformed single colony on LB agar plate to $5 \mathrm{~mL} \mathrm{LB}$ medium as seed culture. When cell growth reached stationary phase, $200 \mu \mathrm{L}$ of seed culture was re-inoculated to
$100 \mathrm{~mL}$ LB medium in a $250 \mathrm{~mL}$ flask. The cultures were then induced with $1.0 \mathrm{mM}$ IPTG when $\mathrm{OD}_{600}$ reached 0.4-0.6 and allowed to grow for an additional $10 \mathrm{~h}$ at $30{ }^{\circ} \mathrm{C}$ and $200 \mathrm{rpm}$. The effects of induction $\mathrm{OD}_{600}$, temperature and IPTG concentration on the whole-cell activity were investigated.

The biosynthesis of $\mathrm{CHOP}$ by whole-cell biotransformation For the production of $\mathrm{CHOP}$, the whole-cell biotransformation was carried out in a $50 \mathrm{~mL}$ flask with $20 \mathrm{~mL}$ reaction broth containing resting cells $\left(\mathrm{OD}_{600}=10\right), 200 \mathrm{mM}$ PBS buffer $(\mathrm{pH}=6.5), 10 \mathrm{~g} / \mathrm{L}$ L-proline, $13 \mathrm{~g} / \mathrm{L} \alpha-\mathrm{KG}$, $5.0 \mathrm{mM} \mathrm{Fe}{ }^{2+}$, and $1.7 \mathrm{mM} \mathrm{L}$-ascorbate. The reaction was performed at a temperature of $30{ }^{\circ} \mathrm{C}$ and stirred at $200 \mathrm{rpm}$. At the specific intervals of the reaction, samples were taken to measure the concentration of L-proline, $\alpha-K G$ and CHOP. To identify the factors limiting catalytic efficiency, the effects of surfactant solutions $(0.5 \%$ Tween 80 and $0.5 \%$ Triton X-100), $\mathrm{pH}, \mathrm{Fe}^{2+}$ concentration, and substrate concentration were investigated.

To supply $\alpha-K G$ economically, an engineered strain BL21/pET28a-LOGX that could convert L-glutamate to $\alpha-K G$ was coupled with CHOP producing strain. The two strains were collected, washed twice, and resuspended in a reaction mixture containing $200 \mathrm{mM}$ PBS (pH 6.5), $10 \mathrm{~g} / \mathrm{L} \mathrm{L}$-proline, $3.0 \mathrm{mM} \mathrm{Fe}{ }^{2+}$, and $1.7 \mathrm{mM} \mathrm{L}$-ascorbate. 5,10 or $20 \mathrm{~g} / \mathrm{L}$ of L-glutamate was supplemented to identify the suitable substrate ratio. In this coupling system, the final cell concentration was about 10 of $\mathrm{OD}_{600}$ for each strain. The reaction was carried out in a $50 \mathrm{~mL}$ 
flask with a work volume of $15 \mathrm{~mL}$ at $30^{\circ} \mathrm{C}$ and $200 \mathrm{rpm}$. Samples were taken at the specific intervals to measure the concentration of $\mathrm{CHOP}, \mathrm{L}$-proline, L-glutamate, and $\alpha-K G$.

\section{Analytical methods}

Cell growth was monitored by measuring absorbance at $600 \mathrm{~nm}$ with BioMate 3S UV-visible spectrophotometer (Thermo). Aqueous concentrations of L-proline and CHOP were analyze by high-performance liquid chromatography (HPLC) system (Agilent 1100 series, Santa Clara, CA) equipped with a evaporative light scattering detector (ELSD) and a Prevail C18 column $(250 \times 4.6 \mathrm{~mm}, 5 \mu \mathrm{m}$, Bio-Rad, Hercules, CA, USA $)$. The column temperature was maintained at $28.5{ }^{\circ} \mathrm{C}$. The mobile phase was consisted of $0.7 \%(\mathrm{v} / \mathrm{v})$ aqueous trifluoroacetic acid and $0.0653 \%(\mathrm{v} / \mathrm{v})$ aqueous heptafluorobutyric acid and supplied at a flow rate of $1.0 \mathrm{~mL} / \mathrm{min}$. Analysis of $\alpha-K G$ and $\mathrm{L}$-glutamate concentration was performed by a HPLC system (Agilent1290, Santa Clara, CA) equipped with the Agilent G1362A refractive index detector. The samples were separated on an Aminex HPX-87H ion-exchange column (Bio-Rad, Hercules, CA, USA) operating at $55^{\circ} \mathrm{C}$ with $5 \mathrm{mM} \mathrm{H}_{2} \mathrm{SO}_{4}$ as the mobile phase $(0.6 \mathrm{~mL} / \mathrm{min})$. The intracellular $\mathrm{CHOP}$ amount was analyzed by Waters time-of-flight mass spectrometry (GC-TOF-MS), which was equipped with a DB-5 fusedsilica capillary column $(30 \mathrm{~m} \times 0.25 \mathrm{~mm}$ i.d., flm thickness $0.25 \mu \mathrm{m}$, J\&W Scientifc, Folsom, CA). Quenching, metabolite extraction and derivatization were performed according to methods described previously [15]. The area of $\mathrm{CHOP}$ peak was then normalized to that of internal standard in the same chromatogram.

\section{Results}

To develop an efficient whole-cell biotransformation system for microbial production of CHOP, it is critical to identify the key factors limiting whole-cell activity. Here, the factors including L-proline cis-4-hydroxylase expression, L-proline degradation by endogenous metabolism, L-proline uptake, and $\alpha$-ketoglutarate (KG) supply were investigated.

\section{The expression of L-proline cis-4-hydroxylase in E. coli}

To obtain the whole-cell biocatalysts that could convert L-proline to CHOP, SmP4H from S. meliloti was cloned into plasmid pETDuet-1 and transformed into different E. coli host cells. As E. coli BL21/pETDuet-SmP4H exhibited higher catalytic activity than E. coli TransB/ pETDuet-SmP4H (Fig. 2a), E. coli BL21(DE3) was selected as the host cell. However, obvious SmP4H synthesis was not observed in the recombinant E. coli BL21/ pETDuet-SmP4H according to the SDS-PAGE analysis.
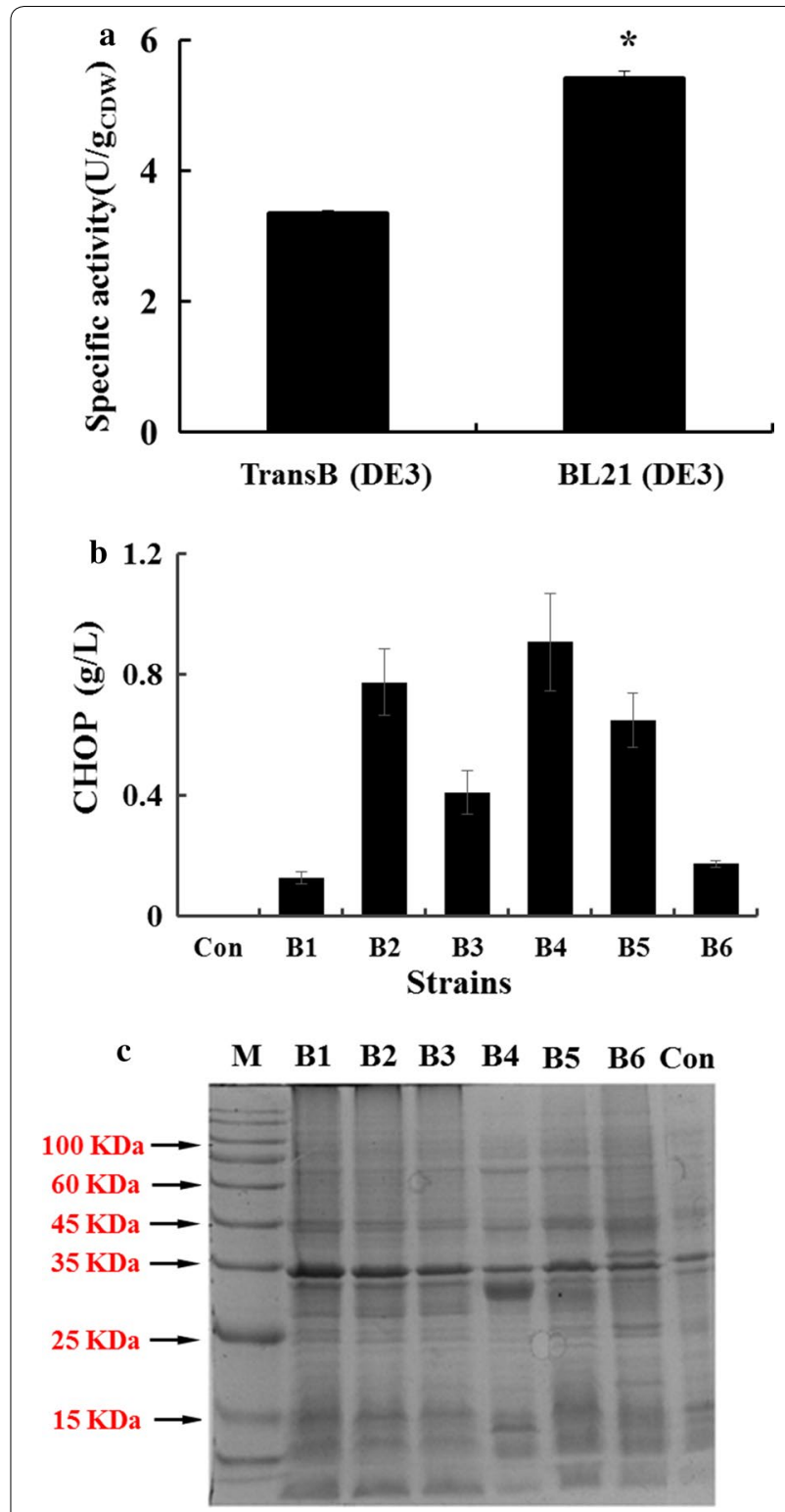

Fig. 2 Optimizing the expression of L-proline cis-4-hydroxylase in E. coli. a CHOP production with different host cells. SmP4H was expressed in E. coli BL21(DE3) and TransB (DE3) respectively; b CHOP production from the resting cells of E. coli BL21(DE3) carrying different expression plasmids. c SDS-PAGE results for the soluble fractions of cell extracts of E. coli BL21(DE3) carrying different expression plasmids. Con, BL21(DE3); B1, BL21/pACYCDuet-1-SmP4H; B2, BL21/ pCDFDuet-1-SmP4H; B3, BL21/pRSFDuet-SmP4H; B4, BL21/pET28aSmP4H; B5, BL21/pET22b-SmP4H; B6, BL21/pETDuet-1 SmP4H

Thus, the different expression systems were employed to optimize SmP4H synthesis (Additional file 1: Figure S1). The highest activity was observed in the resting cells of BL21/pET28a-SmP4H (Fig. 2b), which exhibited the highest SmP4H expression level by the SDS-PAGE analysis (Fig. 2c). These results indicated that the expression 
hosts and expression system had a significant effect on the whole-cell activity and thus influenced CHOP production.

The effects of cultivation conditions including induction temperature, IPTG concentration and induction $\mathrm{OD}_{600}$ on whole-cell activity of BL21/pET28a-SmP4H were also evaluated. Results showed that the decreasing induction temperature significantly increased whole-cell activity (Additional file 1: Figure S2). CHOP titer under condition of $20{ }^{\circ} \mathrm{C}$ was twofold higher than that under condition of $30^{\circ} \mathrm{C}$. Various IPTG concentrations ranging from 0.1 to $1 \mathrm{mM}$ showed little effect on whole-cell activity. The optimal induction time was observed at the early exponential phase (Additional file 1: Figure S2).

\section{The effect of endogenous L-proline degradation on CHOP production}

To evaluate whether L-proline degradation in E. coli BL21/pET28a-SmP4H affects the CHOP production, we employed the CRISPRi technology to repress the expression of putA gene (encoding L-proline dehydrogenase). To carry out the CRISPRi platform in E. coli, a heterologous gene coding for a red fluorescent protein was targeted by CRISPRi to confirm the successful utility of CRISPRi for gene repression (data not shown). When putA gene was targeted by CRISPRi, the PutA activity in the crude extracts of BL21/pET28a-SmP4H+anti-putA was reduced by $70 \%$ compared to that in BL21/pET28aSmP4H (Fig. 3a). The L-proline degradation in the wholecell of BL21/pET28a-SmP4H+anti-putA was also largely repressed as we expected (Fig. $3 \mathrm{~b}$ ). The CHOP production was increased to $3.9 \mathrm{~g} / \mathrm{L}$ with a yield of $64.6 \%$ by repressing putA after bioconversion of $36 \mathrm{~h}$, while only $2.3 \mathrm{~g} / \mathrm{L}$ of CHOP was produced in the whole-cell of BL21/pET28a-SmP4H with a yield of $45.3 \%$ (Fig. 3c). These results indicated that the L-proline degradation by endogenous metabolism is one of the limited factors influencing $\mathrm{CHOP}$ production from $\mathrm{L}$-proline.

\section{The effect of L-proline uptake on CHOP production}

In $E$. coli, L-proline is mainly transported into cells through a $\mathrm{Na}^{+} / \mathrm{L}$-proline transporter encoded by gene putP [16]. To examine whether L-proline uptake over cellular membranes was limited and thus affected biocatalyst efficiency, cells of BL21/pET28a$\mathrm{SmP} 4 \mathrm{H}$ were permeabilized using Tween 80 or Triton $\mathrm{X}-100$. Interestingly, the $\mathrm{CHOP}$ formation by permeabilized cells were all significantly higher than that by resting cells (Fig. 4a), suggesting that substrate uptake was limited during CHOP production. To address this problem, we modified the putP expression level in the strain BL21/pET28a-SmP4H. As shown in Fig. 4b, when gene putP was overexpressed with plasmid pACYCDuet-1,

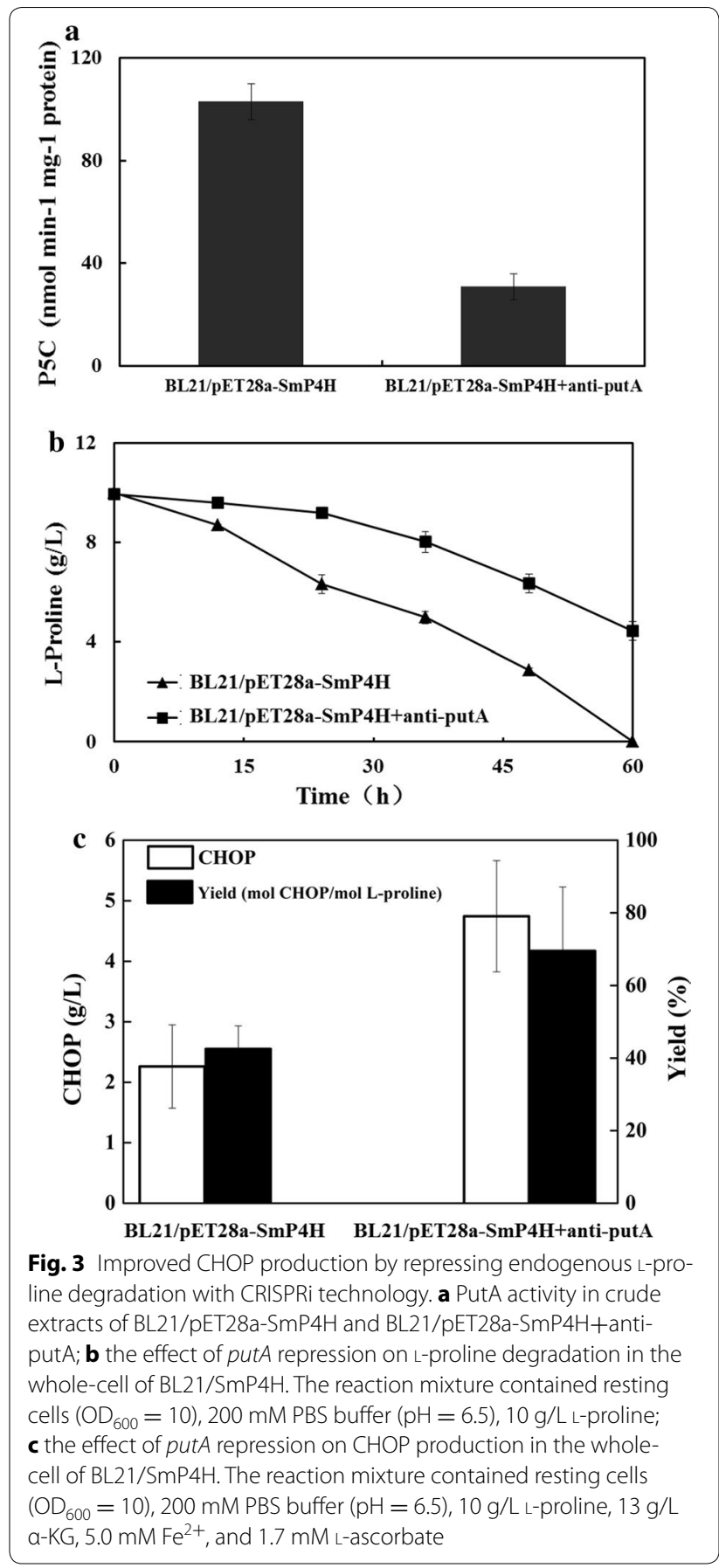

the CHOP production was improved to 2.4 from $2.0 \mathrm{~g} / \mathrm{L}$. With a higher copy number plasmid pET28a, the CHOP production was significantly improved, which could reach $3.6 \mathrm{~g} / \mathrm{L}$. Meanwhile, the intracellular $\mathrm{CHOP}$ accumulation was also evaluated. As the results illustrated in Fig. 4c, the higher level of intracellular CHOP was detected in the two putP overexpressing strains, while the recombinant strain BL21/pET28a-SmP4H-putP accumulated the highest level of intracellular CHOP. The effect 

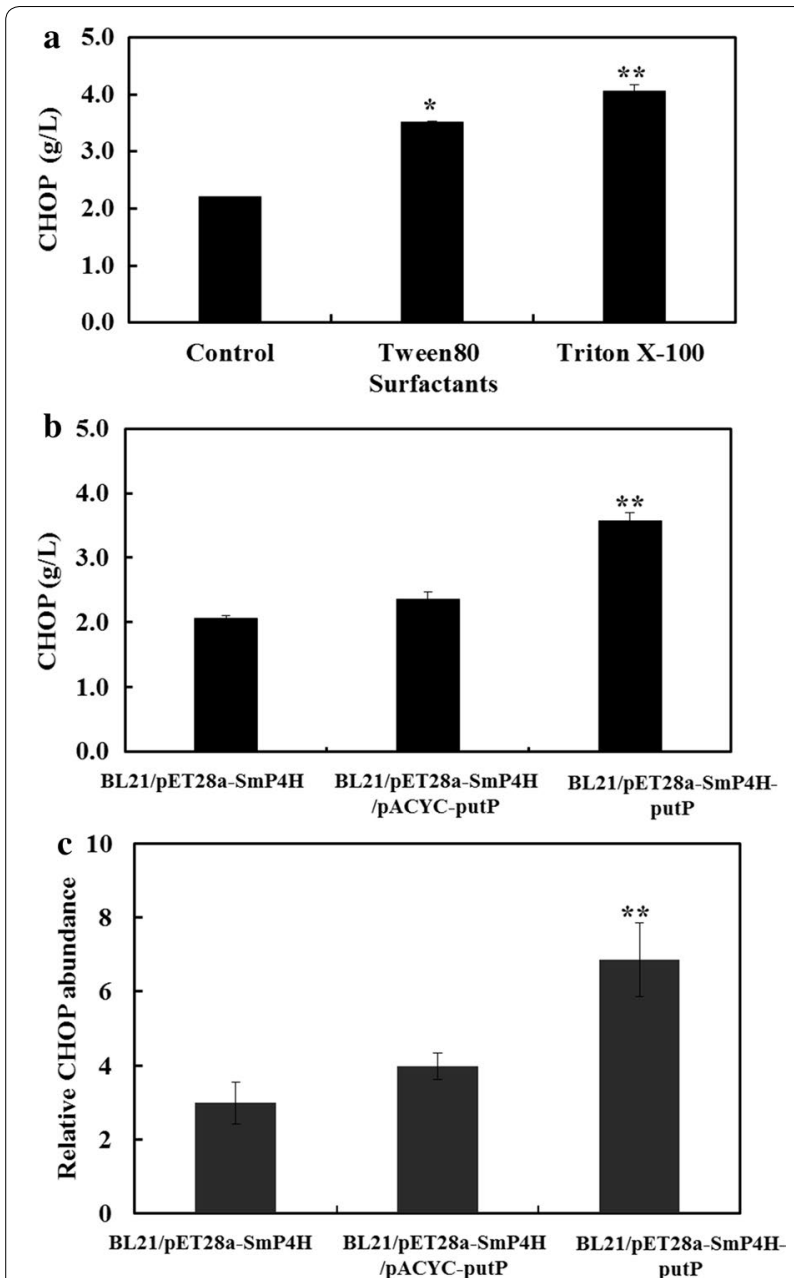

Fig. 4 The effect of L-proline uptake on CHOP production. a The CHOP production by permeabilized cells of E. coli BL21/pET28a$\mathrm{SmP} 4 \mathrm{H} ; \mathbf{b}$ the effect of overexpressing putP in E. coli BL21/pET28a$\mathrm{SmP} 4 \mathrm{H}$ with different plasmids on $\mathrm{CHOP}$ production; $\mathbf{c}$ the effect of overexpressing putP in E. coli BL21/SmP4H with different plasmids on intracellular CHOP level. The relative $\mathrm{CHOP}$ abundance was calculated by normalizing the peak area of $\mathrm{CHOP}$ with internal standard. Results are expressed as mean \pm standard error of the mean $(n=3)$. Significance levels of Students $t$ test: ${ }^{*} P<0.01,{ }^{* *} P<0.001$

of overexpressing putP on intracellular CHOP formation was well consistent with extracellular $\mathrm{CHOP}$ production. These results further reflected the L-proline uptake limitation in the whole-cell transformation system for CHOP production.

\section{The production of CHOP by the optimized whole-cell biocatalysts}

As mentioned above, weakened L-proline degradation and enhanced L-proline uptake could positively influence the whole-cell catalytic performance for $\mathrm{CHOP}$ production. To investigate if these two effects are additive, $E$. coli strain BL21/pET28a-SmP4H-putP+anti-putA was
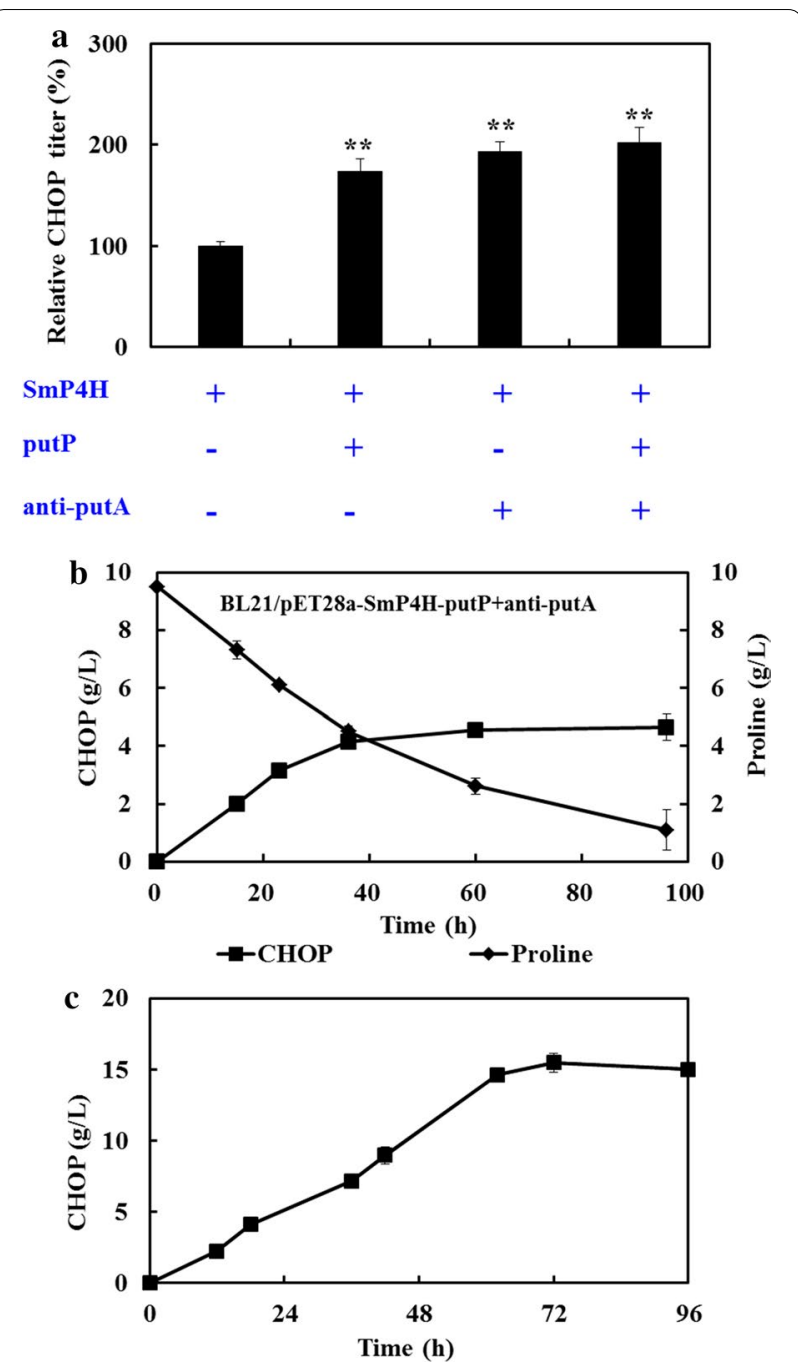

Fig. 5 The CHOP production with the optimum recombinant $E$. coli. a The additive effect of repressing putA and overexpressing putP on $\mathrm{CHOP}$ production. Results are expressed as mean standard error of the mean $(n=3)$. Significance levels of Students $t$ test: ${ }^{*} P<0.01$, ${ }^{*} \mathrm{P}<0.001 ; \boldsymbol{b}$ the CHOP production and L-proline consumption by resting cells of E. coli BL21/pET28a-SmP4H-putP+anti-putA; $\mathbf{c}$ The synthesis of CHOP by the resting cells of BL21/pET28a-SmP4HputP+anti-putA under the optimal reaction condition with a fed batch strategy

constructed by co-transformation of plasmid pET28aSmP4H-putP, pACYC-dCas9, and pCDF-anti-putA. As the results illustrated in Fig. $5 \mathrm{a}$, the final $\mathrm{CHOP}$ titer produced by the whole-cell of BL21/pET28a-SmP4H-putP and BL21/pET28a+anti-putA represented a 1.7 - and 1.9-fold increase respectively compared with that by the whole-cell of BL21/pET28a-SmP4H. The highest CHOP production was observed when the strain BL21/pET28aSmP4H-putP+anti-putA was used as whole-cell biocatalysts, which is 2.0-fold higher than that by the whole-cell of BL21/pET28a-SmP4H. It indicated that the beneficial 
effects of overexpressing putP and repressing putA on whole-cell activity were slightly additive.

Finally, the recombinant strain BL21/pET28a-SmP4HputP+anti-putA was cultivated and collected to perform whole-cell biotransformation process for $\mathrm{CHOP}$ production. After bioconversion of $36 \mathrm{~h}, 4.2 \mathrm{~g} / \mathrm{L} \mathrm{CHOP}$ was produced from $10 \mathrm{~g} / \mathrm{L} \mathrm{L}$-proline (Fig. $5 \mathrm{~b}$ ). The reaction conditions were also investigated. As shown in Additional file 1: Figure S3, the reaction $\mathrm{pH}$ significantly affected the whole-cell catalytic activity, which reached the highest level under the condition of $\mathrm{pH}$ 6.5. The concentration of $\mathrm{Fe}^{2+}$ had a minor effect. In addition, a substrate inhibition might exist in our whole-cell system when the L-proline concentration was above $10 \mathrm{~g} / \mathrm{L}$. With a fed-batch strategy, total CHOP accumulation reached a titer of approximately $15.6 \mathrm{~g} / \mathrm{L}$ (Fig. 5c), which was the highest concentration reported thus far.

\section{The development of two-strain coupling whole-cell system to produce $\mathrm{CHOP}$}

In the whole-cell system for CHOP production, the extra supplement of $\alpha-K G$ was necessary as co-substrate. However, the high cost of $\alpha-K G$ might limit the potential for the industrial CHOP production. To address this problem, an engineered strain BL21/LGOX that expressed L-glutamate oxidase from S. ghanaensis was developed to synthetize $\alpha-K G$ from $L$-glutamate. This strain was then coupled with strain BL21/pET28a-SmP4H-putP+anti-putA as the cocatalysts for $\mathrm{CHOP}$ production (Fig. 6a). As we expected, the product $\mathrm{CHOP}$ was successfully detected. Then, varied concentration ratio of L-proline and L-glutamate was carried out to investigate their effects on $\mathrm{CHOP}$ production. Under the condition of $10 \mathrm{~g} / \mathrm{L} \mathrm{L}$-proline, $2.2 \mathrm{~g} / \mathrm{L}$ of CHOP was produced after the addition of $5 \mathrm{~g} / \mathrm{L}$ L-glutamate (Fig. 6b), while the CHOP titer reached $2.5 \mathrm{~g} / \mathrm{L}$ when L-glutamate concentration was increased to $10 \mathrm{~g} / \mathrm{L}$ (Fig. 6c). When the two-strain coupling system was supplemented with $10 \mathrm{~g} / \mathrm{L} \mathrm{L}$-proline and $20 \mathrm{~g} / \mathrm{L} \mathrm{L}$-glutamate, the CHOP production could be improved to $3.9 \mathrm{~g} / \mathrm{L}$. Meanwhile, the accumulation of $\alpha$-KG was observed (Fig. $6 \mathrm{~d}$ ). Under this condition, $13.5 \mathrm{~g} / \mathrm{L}$ of $\mathrm{CHOP}$ could be accumulated with a fed-batch strategy (Fig. 6e). The titer was similar with that produced by the single strain system, indicating the potential application of our two-strain coupling system for the production of L-proline hydroxylase derived compounds. In addition, we found that a negligible amount of succinate as detected in the whole-cell system (Fig. 6), probably due to the endogenous degradation.

\section{Discussion}

Hydroxy amino acids are important intermediates in the industrial synthesis of valuable chiral compounds [1719]. For their microbial production, several free amino acid-hydroxylating enzymes have been characterized [20]. In our current work, to develop an efficient wholecell process for $C H O P$ production, the recombinant $E$. coli expressing L-proline cis-3-hydroxylase was constructed, and factors associated with microbial physiology were focused on to identify the process limitations that impact the efficiency of biocatalytic transformation.

Initially, to explore the expression system suitable for SmP4H expression, two different $E$. coli host strains and six expression plasmids were employed. The engineered strain BL21/pET28a-SmP4H, which showed highest $\mathrm{SmP} 4 \mathrm{H}$ expression level, exhibited the highest biocatalytic activity, while SmP4H synthesis in other system was poor (Fig. 2). These results suggested a SmP4H synthesis limitation in E. coli. Several other microbial physiology related factors such as substrate uptake and degradation were then investigated. To reduce the L-proline degradation by host intrinsic enzymes, the expression of gene putA in BL21/pET28a-SmP4H was repressed using a CRISPRi technology. Its beneficial effect on CHOP production and yield suggested that L-proline hydroxylation process was limited by intracellular L-proline availability (Fig. 3). In addition, substrate transporters have been reported as excellent targets for strain improvement in the whole-cell system [21]. By using permeabilized cells, the improved biocatalytic activity inferred the limitations of the $\mathrm{L}$-proline uptake. When a $\mathrm{Na}^{+} / \mathrm{L}$-proline permease putP was overexpressed in the $\mathrm{SmP4H}$ containing strain BL21/pET28a-SmP4H, the intracellular and extracellular CHOP accumulation were all positively affected correlated with the increased putP expression level. These results further indicate that factors affecting intracellular L-proline availability were critical for improving biocatalysts activity in the whole-cell bioconversion system for CHOP production, which was consistent with the conclusion from the previous studies that L-proline uptake and competition of THOP formation from L-proline by L-proline-4-hydroxylase with L-proline catabolism were the key factors limiting biocatalytic efficiency for THOP production [22]. However, the additive effect of overexpressing putP and repressing putA was slight (Fig. 5). Increasing the extracellular L-proline concentration could not substantially enhance CHOP production. These results suggested that engineering strategies aiming at further improving $\mathrm{SmP} 4 \mathrm{H}$ synthesis and catalytic properties might be promising for $\mathrm{CHOP}$ biosynthesis.

For the biocatalytic process that utilizes $\alpha$-KG dependent dioxygenases, $\alpha-K G$ was required as a co-substrate. The efficiency of $\alpha-K G$ dependent biotransformation in resting cells has been reported to be limited by the $\alpha$-KG supply in the previous study [10, 22, 23]. In our work, the exogenous supply of $\alpha-K G$ was carried out as an alternative method to solve this problem. However, it 

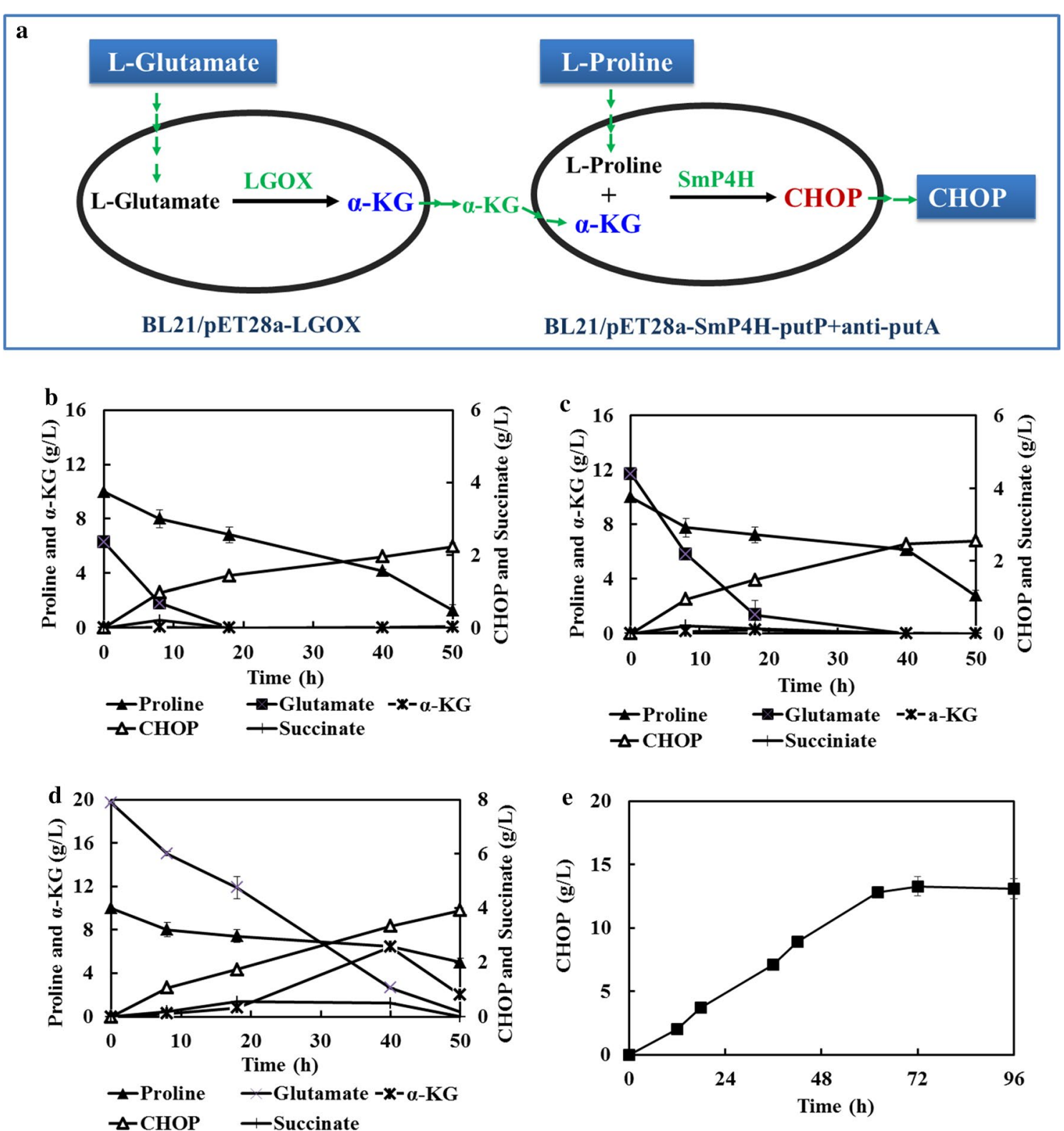

Fig. 6 The two strain coupling whole-cell system to supply a-KG from L-glutamate to produce CHOP. $\mathbf{a}$ Scheme of CHOP synthesis catalyzed by the co-cultures of recombinant E. coli BL21/pET28a-LGOX and E. coli BL21/pET28a-SmP4H-putP+anti-putA; $\mathbf{b}$ the variance of CHOP, L-proline, L-glutamate and a-KG under the condition of $5 \mathrm{~g} / \mathrm{L}$ L-glutamate and $10 \mathrm{~g} / \mathrm{L} \mathrm{L-proline;} \mathbf{c}$ the variance of CHOP, L-proline, L-glutamate and a-KG under the condition of $10 \mathrm{~g} / \mathrm{L} \mathrm{L}$-glutamate and $10 \mathrm{~g} / \mathrm{L} \mathrm{L}$-proline; $\mathbf{d}$ the variance of CHOP, L-proline, L-glutamate and a-KG under the condition of $20 \mathrm{~g} / \mathrm{L}$ L-glutamate and $10 \mathrm{~g} / \mathrm{L}$ L-proline. e The CHOP production with a fed-batch strategy under the condition of $20 \mathrm{~g} / \mathrm{L}$ L-glutamate and $10 \mathrm{~g} / \mathrm{L} \mathrm{L-proline}$

was a cost burden to the bioconversion process. Thus, the solution of $\alpha$-KG supply problem has important significance for developing an economically viable process. The major strategy that has been already employed was to reconstitute the tricarboxylic acid (TCA) cycle of $E$. coli to force and increase $\alpha-K G$ flux through $\alpha-K G$ dependent hydroxylase [23, 24]. However, the $\alpha-K G$ production via these approaches remained far below that required for industrial applications. L-Glutamate was thought as a promising starting material for $\alpha-K G$ production since it was an industry at overcapacity [25]. Along this line of consideration, we developed a novel strategy to relieve $\alpha-K G$ supply limitation during CHOP production process with a two-strain coupling system, where one of the strains expressing LGOX was constructed to supply $\alpha-K G$ from L-glutamate. Excitingly, CHOP was successfully synthesized in our two-strain coupling system (Fig. 6). At a lower level of L-glutamate concentration, the $\mathrm{CHOP}$ 
titer was also limited by the lack of $\alpha-K G$, probably due to the degradation of $\alpha-K G$ by endogenous metabolism (Additional file 1: Figure S4). With excessive L-glutamate supplementation, the CHOP titer could reach $3.9 \mathrm{~g} / \mathrm{L}$, a similar level with the extra addition of $\alpha-K G$. To improve the efficiency of this system, engineering efforts to reduce $\alpha-K G$ consumption would be focused on in the future studies.

Our work clearly demonstrated the factors that could influence biocatalytic performance in a whole-cell bioconversion system for $\mathrm{CHOP}$ production. Through artificially engineering host phenotypes, the biocatalysts efficiency has been largely improved. Meanwhile, with a two-strain coupling system, the whole-cell process was more economical. Finally, $13.5 \mathrm{~g} / \mathrm{L}$ of $\mathrm{CHOP}$, the highest titer reported so far, was obtained in our work. To our knowledge, this is the first report that $E$. coli strain was artificially engineered to product CHOP with a whole-cell biotransformation process, especially with a two-strain coupling whole-cell process. However, this titer was remained far below that required for industrialization application. To further investigate this issue, the capacity of the cell needs to be utilized efficiently by optimized L-proline cis-4-hydroxylase expression with some engineering approaches, i.e., using "trial and error" approaches and modifying the structure of the $5^{\prime}$ end of the mRNA $[26,27]$. In addition, the host strain will be further metabolically engineered to modify characteristics regarding L-proline uptake and $\alpha$-KG consumption.

\section{Conclusions}

Identifying limitations for the whole-cell SmP4H-catalyzed process was demonstrated as the first step toward process development for $\mathrm{CHOP}$ production. A strong interference of microbial catalytic activity with the SmP4H expression, and L-proline uptake and metabolism was demonstrated in our study. Taken together, we have established some engineering strategies to address these limitations by optimizing the expression system, repressing putA gene and overexpressing a L-proline transporter putP. Meanwhile, to reduce the material cost and make the process more economically feasible, a two-strain coupling whole-cell system was developed to address the $\alpha-K G$ supply problem. This work highlights the limitations for $\mathrm{Fe} / \alpha \mathrm{KG}-\mathrm{DO}$ dependent process and provided opportunities for further engineering of recombinant biocatalysts to facilitate process feasibility and improve process economics.

\section{Additional file}

Additional file 1. Additional tables and figures.

\section{Authors' contributions}

WX, OPK and CKQ conceived and designed the experiments. CKQ and PY performed the experiments and analyzed the data. ZBW, FJ and XS contributed to experimental design and also critically revised the manuscript. CKQ and PY wrote the paper. All authors read and approved the final manuscript.

\section{Acknowledgements}

Not applicable.

Competing interests

The authors declare that they have no competing interests.

\section{Availability of data and materials}

The datasets supporting the conclusions of this article are included within the article.

Consent for publication

Not applicable.

\section{Ethics approval and consent to participate}

Not applicable. The manuscript does not report data from humans or animals.

\section{Funding}

This research was financially supported by the National Nature Science Foundation of China (Grant Nos. 21576134, 21606127), "863" program of China (Grant No. 2015AA021005) and the National Key Research and Development Program of China (Grant No. 2016YFA0204300).

\section{Publisher's Note}

Springer Nature remains neutral with regard to jurisdictional claims in published maps and institutional affiliations.

Received: 30 August 2017 Accepted: 12 November 2017

Published online: 22 November 2017

References

1. Chubukov V, Mukhopadhyay A, Petzold C, Keasling J. Synthetic and systems biology for microbial production of commodity chemicals : from target selection to scale-up. npj Syst Biol Appl. 2016;16009:1-11. http:// www.nature.com/articles/npjsba20169.

2. Becker J, Wittmann C. Advanced biotechnology: metabolically engineered cells for the bio-based production of chemicals and fuels, materials, and health-care products. Angew Chemie Int Ed. 2015;54:3328-50.

3. Borodina I, Nielsen J. Advances in metabolic engineering of yeast Saccharomyces cerevisiae for production of chemicals. Biotechnol J. 2014;9:609-20.

4. Becker J, Wittmann C. Bio-based production of chemicals, materials and fuels-Corynebacterium glutamicum as versatile cell factory. Curr Opin Biotechnol. 2012;23:631-40.

5. Bach TMH, Hara R, Kino K, Ohtsu I, Yoshida N, Takagi H. Microbial production of $\mathrm{N}$-acetyl cis-4-hydroxy-L-proline by coexpression of the rhizobium L-proline cis-4-hydroxylase and the yeast $\mathrm{N}$-acetyltransferase Mpr1. Appl Microbiol Biotechnol. 2013;97:247-57. https://doi.org/10.1007/ s00253-012-4204-z.

6. Mai Hoa BT, Hibi T, Nasuno R, Matsuo G, Sasano Y, Takagi H. Production of $\mathrm{N}$-acetyl cis-4-hydroxy-L-proline by the yeast $\mathrm{N}$-acetyltransferase Mpr1. J Biosci Bioeng. 2012;114:160-5. https://doi.org/10.1016/j. jbiosc.2012.03.014.

7. Mueller C, Emmrich J, Jaster R, Braun D, Liebe S, Sparmann G. cisHydroxyproline-induced inhibition of pancreatic cancer cell growth is mediated by endoplasmic reticulum stress. World J Gastroenterol. 2006;12:1569-76.

8. Hara R, Kino K. Characterization of novel 2-oxoglutarate dependent dioxygenases converting L-proline to cis-4-hydroxy-L-proline. Biochem Biophys Res Commun. 2009;379:882-6. https://doi.org/10.1016/j. bbrc.2008.12.158. 
9. Matsuoka T, Furuya K, Serizawa N. Fermentative production of cis4-hydroxy-(I)-proline by Helicocerus oryzae and Acrocylindrium oryzae. Biosci Biotechnol Biochem. 1994;58:1747-8.

10. Theodosiou E, Frick O, Bühler B, Schmid A. Metabolic network capacity of Escherichia coli for Krebs cycle-dependent proline hydroxylation. Microb Cell Fact. 2015;14:108. http://www.microbialcellfactories.com/ content/14/1/108.

11. Shibasaki T, Mori H, Ozaki A. Enzymatic production of trans-4-hydroxyL-proline by regio- and stereospecific hydroxylation of L-proline. Biosci Biotechnol Biochem. 2000;64:746-50. https://doi.org/10.1271/ bbb.64.746.

12. Olaofe OA, Fenner CJ, Gudiminchi R, Smit MS, Harrison STL. The influence of microbial physiology on biocatalyst activity and efficiency in the terminal hydroxylation of $n$-octane using Escherichia coli expressing the alkane hydroxylase, CYP153A6. Microb Cell Fact. 2013;12:8.

13. Qi LS, Larson MH, Gilbert LA, Doudna JA, Weissman JS, Arkin AP, et al. Repurposing CRISPR as an RNA-guided platform for sequence-specific control of gene expression. Cell. 2017;152:1173-83. https://doi. org/10.1016/j.cell.2013.02.022.

14. Wadhawan S, Gautam S, Sharma A. Involvement of proline oxidase (PutA) in programmed cell death of Xanthomonas. PLOS ONE. 2014;9:1-12.

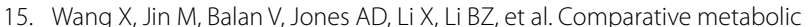
profiling revealed limitations in xylose-fermenting yeast during co-fermentation of glucose and xylose in the presence of inhibitors. Biotechnol Bioeng. 2014;111:152-64.

16. Olkhova E, Raba M, Bracher S, Hilger D, Jung H. Homology model of the $\mathrm{Na}+$ /proline transporter PutP of Escherichia coli and its functional implications. J Mol Biol. 2011;406:59-74.

17. Hibi M, Ogawa J. Characteristics and biotechnology applications of aliphatic amino acid hydroxylases belonging to the $\mathrm{Fe}(\mathrm{II}) / \mathrm{a}$-ketoglutaratedependent dioxygenase superfamily. Appl Microbiol Biotechnol. 2014;98:3869-76. https://doi.org/10.1007/s00253-014-5620-z.

18. Handa T, Yamaguchi K, Sono Y, Yazawa K. Effects of fenugreek seed extract in obese mice fed a high-fat diet. Biosci Biotechnol Biochem. 2005;69:1186-8
19. Smirnov SV, Kodera T, Samsonova NN, Kotlyarova VA, Rushkevich NY, Kivero AD, et al. Metabolic engineering of Escherichia coli to produce (2S, 3R, 4S)-4-hydroxyisoleucine. Appl Microbiol Biotechnol. 2010;88:719-26. https://doi.org/10.1007/s00253-010-2772-3.

20. Hausinger RP. Fe(II)/a-ketoglutarate-dependent hydroxylases and related enzymes. Crit Rev Biochem Mol Biol. 2004;39:21-68. https://doi. org/10.1080/10409230490440541.

21. Kell DB, Swainston N, Pir P, Oliver SG. Membrane transporter engineering in industrial biotechnology and whole cell biocatalysis. Trends Biotechnol. 2015;33:237-46.

22. Falcioni F, Blank LM, Frick O, Karau A, Bühler B, Schmida A. Proline availability regulates proline-4-hydroxylase synthesis and substrate uptake in proline-hydroxylating recombinant Escherichia coli. Appl Environ Microbiol. 2013;79:3091-100. http://aem.asm.org/content/79/9/3091.

23. Lin B, Fan K, Zhao J, Ji J, Wu L, Yang K, et al. Reconstitution of TCA cycle with DAOCS to engineer Escherichia coli into an efficient whole cell catalyst of penicillin G. Proc Natl Acad Sci. 2015;112:9855-9. http://www.pnas. org/content/112/32/9855.abstract.

24. Theodosiou E, Breisch M, Julsing MK, Falcioni F, Bühler B, Schmid A. An artificial TCA cycle selects for efficient a-ketoglutarate dependent hydroxylase catalysis in engineered Escherichia coli. Biotechnol Bioeng. 2017;9999:1-10. https://doi.org/10.1002/bit.26281.

25. Niu P, Dong X, Wang Y, Liu L. Enzymatic production of a-ketoglutaric acid from L-glutamic acid via L-glutamate oxidase. J Biotechnol. 2014;179:5662. https://doi.org/10.1016/j.jbiotec.2014.03.021.

26. Nilsson LO, Mannervik B. Improved heterologous expression of human glutathione transferase A4-4 by random silent mutagenesis of codons in the 5'region. Biochim Biophys Acta. 2001;1528:101-6.

27. Papaneophytou CP, Kontopidis G. Statistical approaches to maximize recombinant protein expression in Escherichia coli: a general review. Protein Expr Purif. 2014;94:22-32.

\section{Submit your next manuscript to BioMed Central and we will help you at every step:}

- We accept pre-submission inquiries

- Our selector tool helps you to find the most relevant journal

- We provide round the clock customer support

- Convenient online submission

- Thorough peer review

- Inclusion in PubMed and all major indexing services

- Maximum visibility for your research

Submit your manuscript at www.biomedcentral.com/submit
O Biomed Central 